International Journal of Environment, Agriculture and Biotechnology
Vol-6, Issue-5; Sep-Oct, 2021

\title{
Nano remediation of Hormonal Endocrine Disrupting Chemicals from Water by Acalypha indica Silver Nanoparticles
}

\author{
Pavani K. Gonnabathula and Momoh A. Yakubu*
}

\begin{abstract}
Department of Environmental and Interdisciplinary Sciences, College of Science, Engineering, and Technology, Texas Southern University, Houston, Texas, USA

*Corresponding Author
\end{abstract}

Received: 12 Apr 2021; Received in revised form: 14 Oct 2021; Accepted: 22 Oct 2021; Available online: 31 Oct 2021 (C2021 The Author(s). Published by Infogain Publication. This is an open access article under the CC BY license (https://creativecommons.org/licenses/by/4.0/).

\begin{abstract}
Endocrine disrupting chemicals are natural or synthetic hormones and can be derived from plastics and pharmaceuticals. They have been found in trace amounts (ppt to ppb) in our water supplies and treated wastewater. They are therefore difficult to remove. We have synthesized silver nanoparticles (AgNP's) using Acalypha indica (A. indica) leaf extracts and used it to treat water samples spiked with hormones to determine efficiency of removal. AgNP's were synthesized and characterized by UV-Vis spectrophotometer, Zetasizer for particle size and zeta potential. To investigate the effects of the AgNP's on the removal of hormonal compounds from water, deionized water was spiked with 6 selected hormones, incubated with synthesized AgNP's and agitation overnight. The effects AgNP's on removal of the hormones was determined by HPLC UV-Vis. Results indicated that the AgNP's synthesized showed absorption spectrum at about $400 \mathrm{~nm}$, confirming formation of AgNP's. The average size and zeta potential analyzed by dynamic light scattering techniques showed the sized to be $132.6 \mathrm{~nm}$ and the Zeta potential as -ve $61.8 \mathrm{mV}$ suggesting higher stability of AgNP's. Nano remediation resulted in changes in peak areas of HPLC chromatograms. Among the selected hormonal compounds, there is a huge reduction of peak areas in the 1 and 5 ppm Diethylstilbestrol $(R t=10.6)$ by $36.68 \%$ and $15.46 \%$; D Norgestrel $(R t=$ 11.6) by $7.97 \%$ and $27.95 \%$; 19 Norethindrone $(R t=8.5)$ by $10.17 \%$ and $0.64 \%$; respectively. There was peak reduction observed for 5 ppm of 17 Alpha ethynyl estradiol $(R t=8.6)$ by $2.33 \%, 1$ ppm Beta Estradiol $(R t=7.6)$ by $10.35 \%$ and Estrone $(R t=9.4)$ by $6.47 \%$. Results indicate that we successfully synthesized AgNP's and demonstrated nano remediation of hormonal compounds from water. This is a novel effort that need further investigation for broader application in water treatment processes and possible scale up production A. indica AgNP's.
\end{abstract}

Keywords - Hormonal Compounds, Endocrine Disruptors, Acalypha indica, Silver Nanoparticles.

\section{INTRODUCTION}

The unbounded presence of hormonal compounds/endocrine disruptor compounds (EDCs) in wastewater has emerged as a threat to aquatic species and humans. The presence of EDC in drinking water has resulted in substantial concerns due to possible toxicological and ecological impacts, with the possibility of introduction of these EDCs into our food chain at trace concentrations. Unfortunately, the conventional wastewater treatment protocols are not able of completely removing these EDCs from water. There is therefore the need to develop effective means to remove them from our environment [Chauhan A, et al 2019]. Remediation is the process of removal of contaminants or pollutants from environment such as soil, water, groundwater, sediments, or air for the general protection of human health and the environment [Ingle AP et al., 2014]. Currently, Environmental remediation is achieved by the use of existing methods like physical, chemical and biological remediation but may not be effective in completely 
removing $(100 \%)$ of toxicants from the environmental media. An alternative approach needs to be developed to be used to have a clean environment. Now the focus has shifted towards nanotechnology for the successful remediation of these persistent pollutants as such provide ample opportunity for effective removal of EDC from environmental matrices [Ingle AP et al., 2014].

Nanotechnology is an emerging technology with many applications. It involves the synthesis and application of materials with dimensions in the range of 1-100 nm [Bar et al., 2009; Rassaei et al., 2008]. Due to the exceptional properties of nanoparticles which possess large surface areas, are mechanically strong, optically active, and are chemically reactive and can be used to trap chemical contaminants. These make them unique and suitable candidates for different applications e.g., nanodevices for use in not only for environmental cleanup but also in biological, physical, biomedical and pharmaceutics.

Plants have been a source of filter for our water resourcesthey line our streams and rivers, populate the riverbanks and spread over the water sheds which serves as reservoir and collection points for run offs and storm water before they are discharged into other bodies of water. The waters from these rivers/streams serves as drinking water for several communities. Plants are rich in several biological resources which make them natural resources for several purposes. Apart from providing shades reducing water evaporation, plants help in filtration processes removing impurities from the water bodies. This is made possible because of the versatility of plant constituents. Plant extract produce a wide range of constituents and chemical compounds with varied properties. These phytochemicals present in plants have been identified to contain such constituents as tannins, saponins, alkaloids, glycosides, steroids, polyphenols etc. [Zhao et al., 2015]. These natural compounds are highly functionalized which make them capable of reacting with other chemicals possibly neutralize and remove impurities. These properties are being exploited in alternative medicine where they have been shown to have the ability of reducing oxidative stress through antioxidant activity [Das RK et al., 2017]. Therefore, these properties could be useful for use in the removal of environmental chemicals and toxicants from water in remediation process. We have synthesized AgNP's using herbal plant extracts. The resulting nanoparticles were used to treat water samples spiked with known concentrations of EDCs and evaluated for effectiveness in their removal. Many novel nanomaterial adsorbents have been developed for increasing the efficiency and adsorption capacities of removing contaminants from wastewater [Sadegh H et al 2017].
A wide variety of physical- chemical approaches are being used for the synthesis of nanoparticles, most of which involve use of harsh chemicals at high temperatures. However, biogenic reduction of metal precursors to produce corresponding synthesis of nanoparticles is ecofriendly, less expensive, free of chemical contaminants for medical and biological application where purity of synthesis of nanoparticles is of major concern [Gurunathan S. et al 2009; Pal S. et al., 2007; Bae E. et al. 2010].

So, in the current study, we synthesized AgNP's by the reduction of silver ions present in the solution of $\mathrm{AgNO}_{3}$ using the leaf extract (aqueous) of Acalypha indica, characterized by DLS and UV-Vis spectrum. The efficacy for nano remediation was evaluated by treatment of water samples spiked with EDCs to determine the ability of the synthesized nano particle in the removal of these compounds.

\section{MATERIALS AND METHODS}

\section{Materials:}

Six hormonal compounds: 17 alpha ethynyl estradiol, beta estradiol, estrone, diethylstilbestrol, 19 norethindrone, and D norgestrel were purchased from Sigma Aldrich, St Louis Missouri, Silver Nitrate 99\% ACS reagent purchased from Acros Organics, Thermo Fisher Scientific New Jersey US. Acalypha indica leaves was freshly collected (Andhra Pradesh, India) and washed individually under the running water and then with Milli-Q (double distilled) water, it was then air dried and milled into fine powder.

\section{A. indica leaf extraction:}

Approximately 10 grams of air-dried A. indica plant extract was boiled in distilled water for $15 \mathrm{~min}$. The extract obtained was filtered through Whatman \#1 filter paper and the filtrate was stored at $2-4{ }^{\circ} \mathrm{C}$ in the refrigerator for further use.

\section{Synthesis of Ag nanoparticles:}

To a baker containing $43 \mathrm{ml}$ of $0.01 \mathrm{M} \mathrm{AgNO}_{3}, 7 \mathrm{ml}$ of $A$. indica extract was added and heat at $80^{\circ} \mathrm{C}$ for 1 hour. The change from pale yellow to dark brown indicated the formation of colloidal silver nanoparticles. Reaction mixture was centrifuged at 14,000 rpm for 15 min [Fig. 1]. Precipitate was washed with distilled water thrice and then with $100 \%$ ethanol twice. Pellet collected and air dried, stored for further experiment and characterization.

\section{Characterization of the synthesized AgNP's:}

Synthesized AgNP's were characterized by various instrumental analyses. The formation of AgNP's (the bio reduction of the $\mathrm{Ag}$ ) was measured by UV-Vis spectrophotometer. The samples were scanned with a 
wavelength of range of 400-600 $\mathrm{nm}$ operated at a resolution of $1 \mathrm{~nm}$ at room temperature $(25 \mathrm{C})$. Zetasizer (ZS 90, Malvern, UK) was employed to measure the particle size and zeta potential of the AgNP's. The synthesized AgNP's were diluted with PBS (0.15 M, pH 7.2). The aliquots were then sampled in dynamic light scattering (DLS) and nanoparticles were then examined for equivalent diameters, size distribution and zeta potential.

Treatment (Rx) of water samples with known concentration of EDCs and removal by synthesized nanoparticles:

Method:

Hormonal compounds selected and used were 17 alpha ethynyl estradiol, beta estradiol, estrone, diethylstilbestrol, 19 norethindrone, and D norgestrel. Stock concentrations of these hormones were prepared by dissolving $10 \mathrm{mg}$ in $10 \mathrm{ml}$ methanol. Each hormonal compound 1 and $5 \mathrm{ppm}$ were added to deionized water and were subjected to treatment by simple conventional overnight incubation on a shaker along with the synthesized silver nanoparticles of A. indica $(10 \mathrm{ppm})$ to check the remediation efficacy on hormones in water. After incubation (continuous shaking), samples were subjected to centrifugation for 10 minutes at $1500 \mathrm{rpm}$, supernatant collected and filtered through 0.25 $\mu \mathrm{m}$ filters into separatory funnel. The filtrate was extracted with 1:1 ratio of dichloromethane and allowed evaporate to under the hood. The residue was reconstituted with $1 \mathrm{ml}$ methanol and analyzed using HPLC UV-Vis.

\section{Instrument:}

Dionex Ultimate 3000 High Performance Liquid Chromatography with UV detector - Ultimate 3000 HPLC comes with an assembly of binary pump, degasser, autosampler and a UV visible detector that can detect any of the four selected wavelengths from $190 \mathrm{~nm}$ to $400 \mathrm{~nm}$ at a time. The binary pump can work in isocratic and gradient mode. Isocratic mode is single solvent line flowing through the column, gradient mode enables to run a combination of two solvents in a programmed time frame. The degasser equipped to the pump helps in purging out air gaps in the solvent line generated during solvent mixing. Ultimate 3000 is suitable for analytical separations of various chemical compounds.

\section{HPLC UV-Vis Conditions:}

Mobile phase: Water: Acetonitrile 50:50

Flow rate: $0.8 \mathrm{ml} / \mathrm{min}$

Wavelength: $230 \mathrm{~nm}$

Column: Supelco Discovery C18, 250 x 4.6mm, $5.0 \mu$

Diluent: Methanol

\section{SUMMARY OF RESULTS AND DISCUSSION}

The average size and zeta potential were analyzed by dynamic light scattering techniques (DLS) and the size values were shown to be $132.6 \mathrm{~nm}$ [Fig. 2]. Zeta potential provides the information about the stability of nanoparticles and surface charge. Zeta potential of the synthesized AgNP's is pictured [Fig. 3], with -ve $61.8 \mathrm{mV}$ charge suggesting higher stability of AgNP's.

UV-vis spectroscopy is commonly used to confirm metallic nanoparticle formation by studying the optical properties which depend particularly on size effect. Basically, the silver nanoparticles exhibit yellowish brown color in aqueous solution due to excitation of surface plasmon vibrations in silver nanoparticles [Krishnaraj et al.,2010]. Fig 4 Shows the UV-Vis spectra recorded from the reaction medium after heating the solution to $80^{\circ} \mathrm{C}$ for 1 hour. Absorption spectra of silver nanoparticles formed in the reaction media has absorbance peak near $400 \mathrm{~nm}$, which confirmed the formation of silver nanoparticles. This is consistent with others who had synthesized silver nanoparticles with $A$. indica [Krishnaraj et al.,2010]

Treatment of water samples with AgNP's of A. indica at $10 \mathrm{ppm}$ concentration by conventional incubation (due to nanoparticles larger surface and adsorption capacities of removing contaminants from wastewater [Sadegh $\mathrm{H}$ et al., 2017] resulted in changes in the peak areas (The size of the peak is proportional to the concentration of the analyte) [Corradini D et al., 1998] as indicated on the chromatograph identified by HPLC analysis (Fig. 5-16). After treatment there is a significant reduction in the peak areas observed in samples treated with A. indica AgNP's. Among the selected hormonal compounds, there is a huge reduction of peak areas of $1 \mathrm{ppm}$ and $5 \mathrm{ppm}$ Diethylstilbestrol $(\mathrm{Rt}=10.6)$ by $36.68 \%$ and $15.46 \%$ respectively following treatment with $10 \mathrm{ppm}$ AgNP's. After treatment peak areas of $1 \mathrm{ppm}$ and $5 \mathrm{ppm}$ of Dnorgestrel $(\mathrm{Rt}=11.6)$ were reduced by $7.97 \%$ and $27.95 \%$ respectively. Treatment reduced peak areas of $1 \mathrm{ppm}$ and 5 ppm 19 norethindrone $(\mathrm{Rt}=8.5)$ by $10.17 \%$ and $0.64 \%$ respectively. There is also a peak area reduction observed for 5 ppm 17 alpha ethynyl estradiol $(\mathrm{Rt}=8.6)$ by $2.33 \%, 1$ ppm beta estradiol $(\mathrm{Rt}=7.6)$ by $10.35 \%$ and estrone $(\mathrm{Rt}=$ 9.4) by $6.47 \%$. These results are summarized in Table.1.

\section{CONCLUSION}

In this study we have successfully synthesized $A$. indica AgNP's using green synthesis method. Characterization 
confirms the synthesis and shows a stable compound with potential for the use in possible remediation as our results shows that treatment of water samples spiked with hormones resulted in significant reduction in the peak area (The size of the peak is proportional to the concentration of the analyte) [Corradini D et al., 1998]. Our results suggest a potential for the use of AgNP's of A. indica in possible remediation to remove low concentration of hormones and other chemicals from the contaminated environment. However, this needs further studies using samples from wastewater treatment plants for example.

\section{ACKNOWLEDGMENTS}

This research was supported by Tittle III Award Number P031B090216; Title III, Part B, Historically Black Graduate Institutions (HBGI) (CFDA No. 84.031B).

\section{REFERENCES}

[1] Bae E, Park HJ, Lee J, Kim Y, Yoon J, Park K. (2010) Bacterial cytotoxicity of the silver nanoparticle related to physicochemical metrics and agglomeration properties. Envion Toxicol Chem. 29(10):2154-2160.

[2] Bar H, Bhui DK, Sahoo GP, Sarkar P, De SP, Misra A. (2009). Green synthesis of silver nanoparticles using latex of Jatropha curcas. Colloids and Surfaces A: Physicochem. Eng. Aspects 339 (2009) 134-139.

[3] Chauhan A, Sillu D, Agnihotri S. (2019). Removal of Pharmaceutical Contaminants inWastewater Using Nanomaterials: A Comprehensive Review. Curr Drug Metab. 2019;20(6):483-505. doi:10.2174/1389200220666181127104812.

[4] Corradini D, Eksteen E (Katz), Eksteen R,Schoenmakers P, Miller N. (1998) Handbook of HPLC. Taylor \& Francis. ISBN $0824794443,9780824794446$.
[5] Das RK, Pachapur VL, Lonappan L. (2017) Biological synthesis of metallic nanoparticles: plants, animals and microbial aspects. Nanotechnology for Environmental Engineering. 2:18. [ISSN 2365-6387].

[6] Gurunathan S, Kalishwaralal K, Vaidyanathan R, Venkataraman D, Pandian SR, Muniyandi J, et al. (2009) Biosynthesis, purification and characterization of silver nanoparticles using Escherichia coli. Colloids Surf B Biointerfaces. 74(1):328-335.

[7] Ingle PA, Seabra AB, Duran N, and Rai M (2014). Nanoremediation: A New and Emerging Technology for the Removal of Toxic Contaminant from Environment. Microbial Biodegradation and Bioremediation.Pages 233250. [ISBN 978-0-12-800021-2]

[8] Krishnaraj C, Jagan EG, Rajasekar S, Selvakumar P, Kalaichelvan PT, Mohan N. (2010). Synthesis of silver nanoparticles using Acalypha indica leaf extracts and its antibacterial activity against water borne pathogens, Colloids Surf. B 76 (2010) 50-56.

[9] Pal S, Tak YK, Song JM. (2007) Does the antibacterial activity of silver nanoparticles depend on the shape of the nanoparticle? A study of the gram-negative bacterium Escherichia coli. Appl Environ Microbiol. 73:1712-1720.

[10] Rassaei L, Sillanpaa M, French RW, Compton RG, Marken F. (2008) Arsenite Determination in Phosphate Media at Electroaggregated Gold Nanoparticle Deposits. Electroanalysis 20(12):1286 - 1292.

[11] Sadegh H, Ali GAM, Gupta VK, Makhlouf ASH, Shahryarighoshekandi R, Nadagouda MN, Sillanpa M, Megiel E. (2017). The role of nanomaterials as effective adsorbents and their applications in wastewater treatment. J Nanostruct Chem 7:1-14.

[12] Zhao Y., Wu Y., Wang M. (2015) Bioactive Substances of Plant Origin. In: Cheung P., Mehta B. (eds) Handbook of Food Chemistry. Springer, Berlin, Heidelberg. pp $967-$ 1008. [ISBN 978-3-642-36605-5].



(A)



(B)

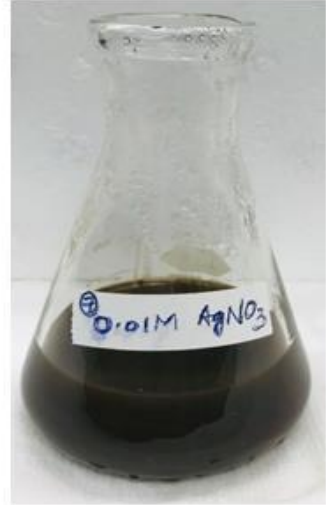

(C)

Fig. 1: Visual identification of silver nanoparticles A) $0.01 \mathrm{M}$ silver B) silver nitrate solution added with plant extract C) brown color change-formation of Ag NP's 


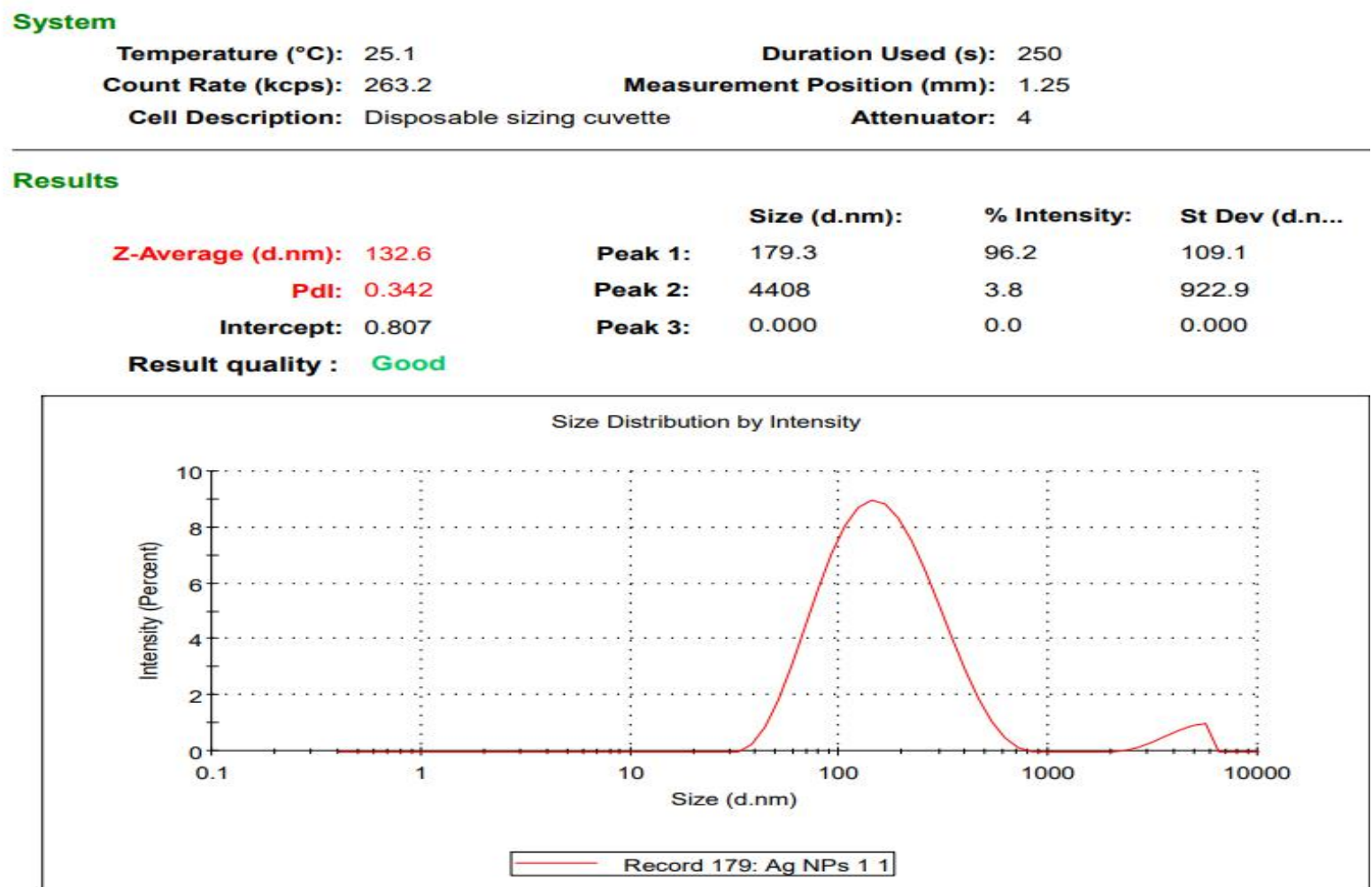

Fig. 2: Size distribution of AgNP's of A. indica measured by the DLS technique



Fig. 3: Zeta potential of AgNP's of A. indica 




Fig. 4: UV-visible Absorption spectrum of silver nanoparticles AgNP1 (Sample 1) and AgNP2 (Sample2)

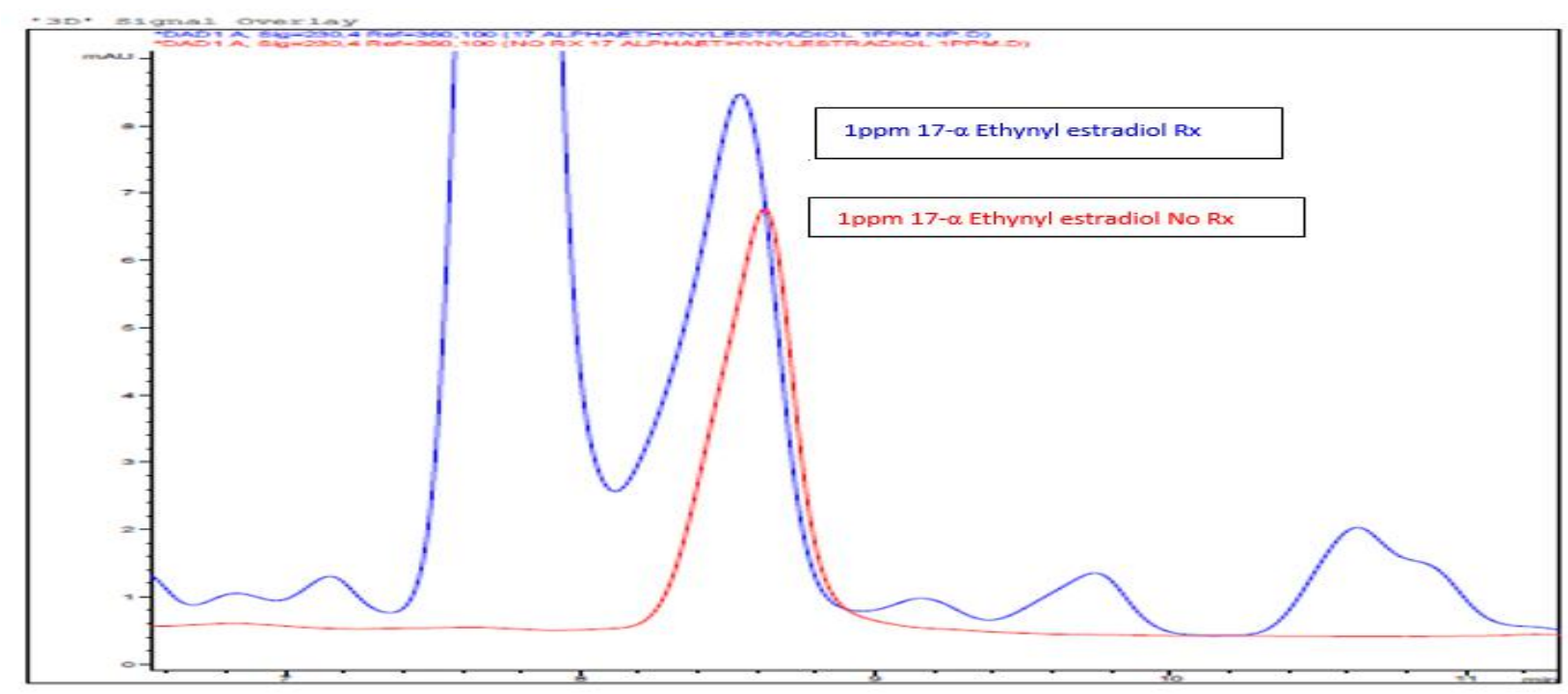

Fig. 5: Overlaid Chromatogram of 1 ppm 17- $\alpha$ Ethynyl estradiol shows changes in peak area with treatment of AgNP's 


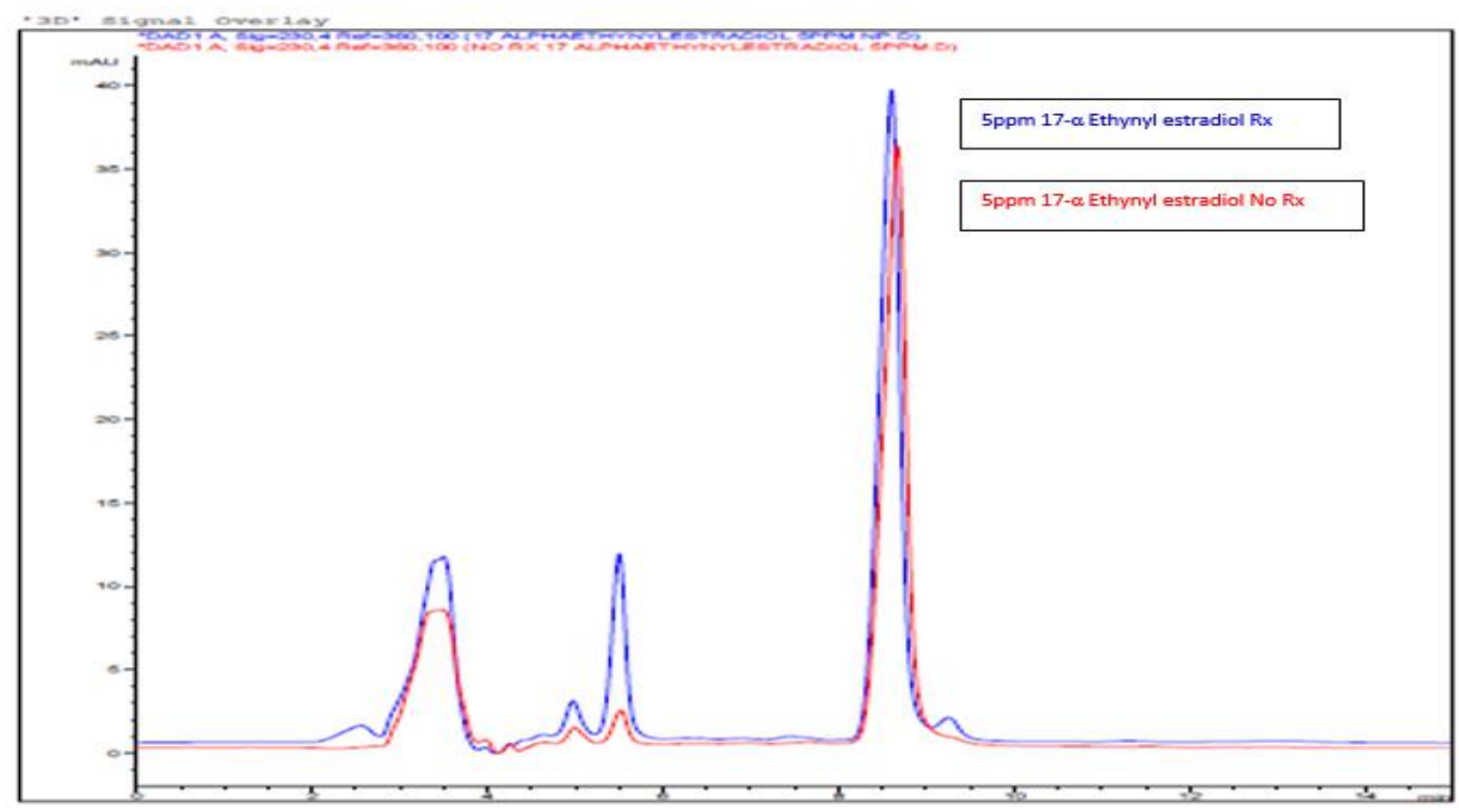

Fig. 6: Overlaid Chromatogram of 5 ppm 17- $\alpha$ Ethynyl estradiol shows changes in peak area with treatment of AgNP's



Fig. 7: Overlaid Chromatogram of 1 ppm 19 Norethindrone shows changes in peak area with treatment of AgNP's 


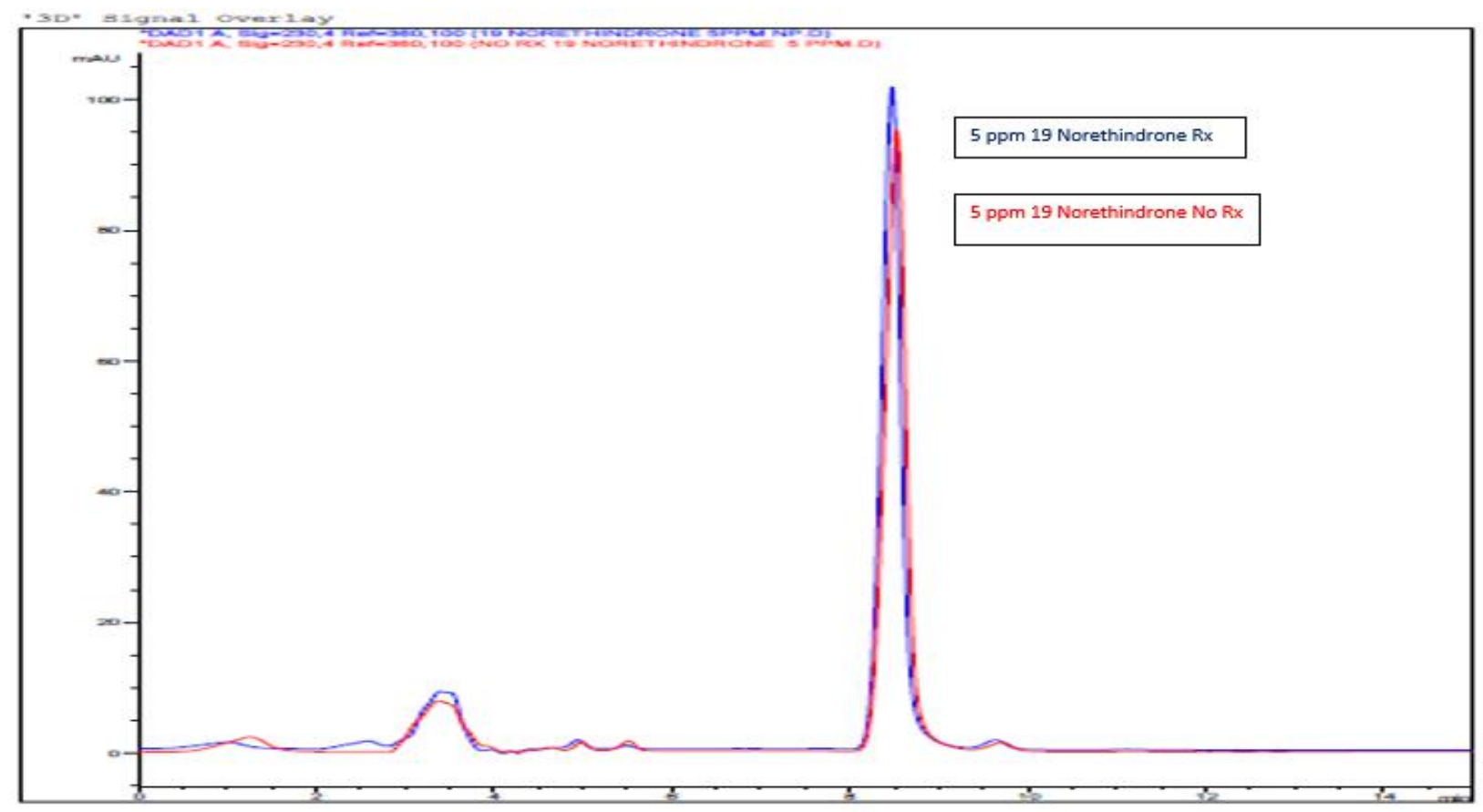

Fig. 8: Overlaid Chromatogram of 5 ppm 19 Norethindrone shows changes in peak area with treatment of AgNP's



Fig. 9: Overlaid Chromatogram of 1 ppm $\beta$-Estradiol shows changes in peak area with treatment of AgNP's 




Fig. 10: Overlaid Chromatogram of 5 ppm $\beta$-Estradiol shows changes in peak area with treatment of AgNP's

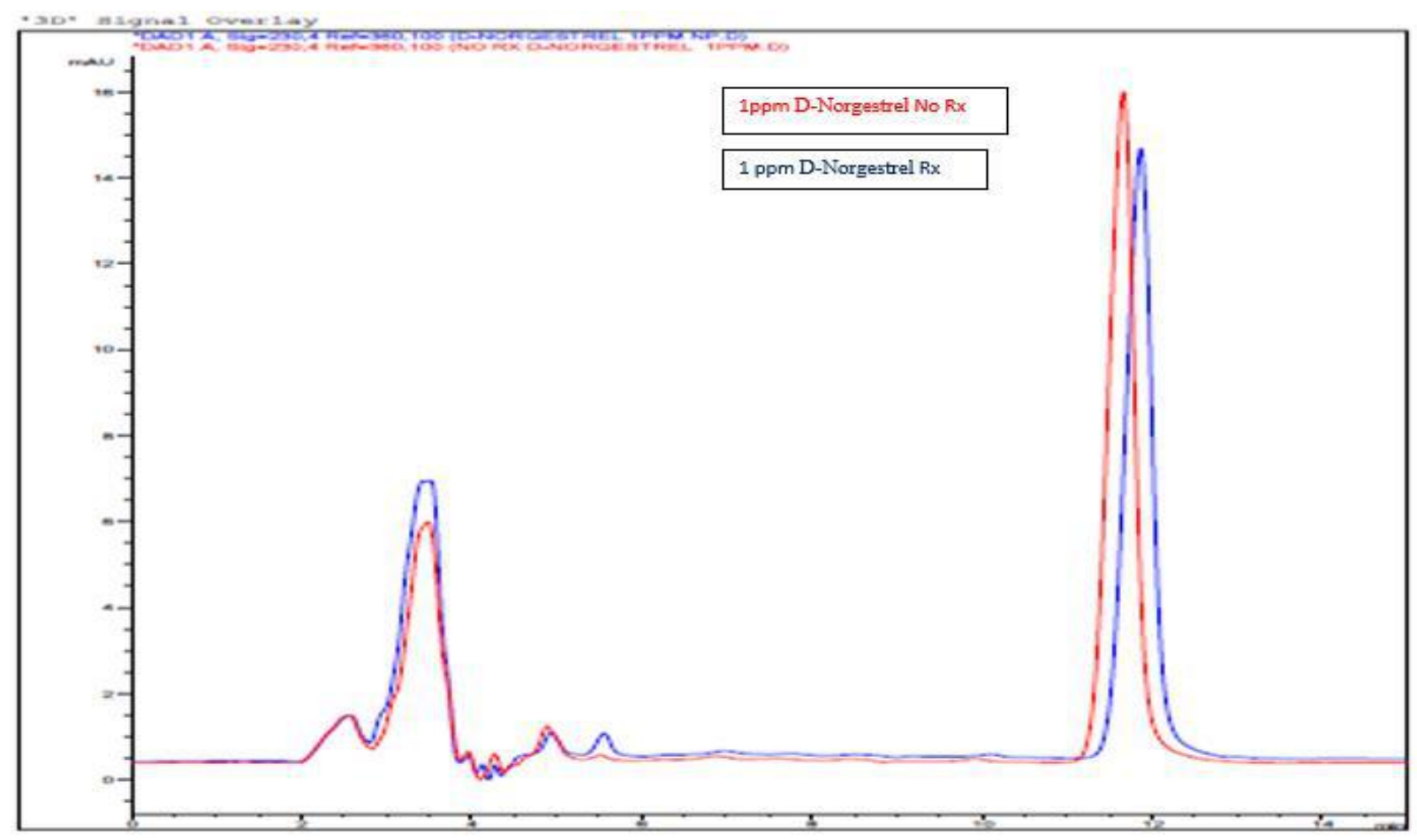

Fig. 11: Overlaid Chromatogram of 1ppm D-Norgestrel shows changes in peak area with treatment of AgNP's 




Fig. 12: Overlaid Chromatogram of 5 ppm D-Norgestrel shows changes in peak area with treatment of AgNP's

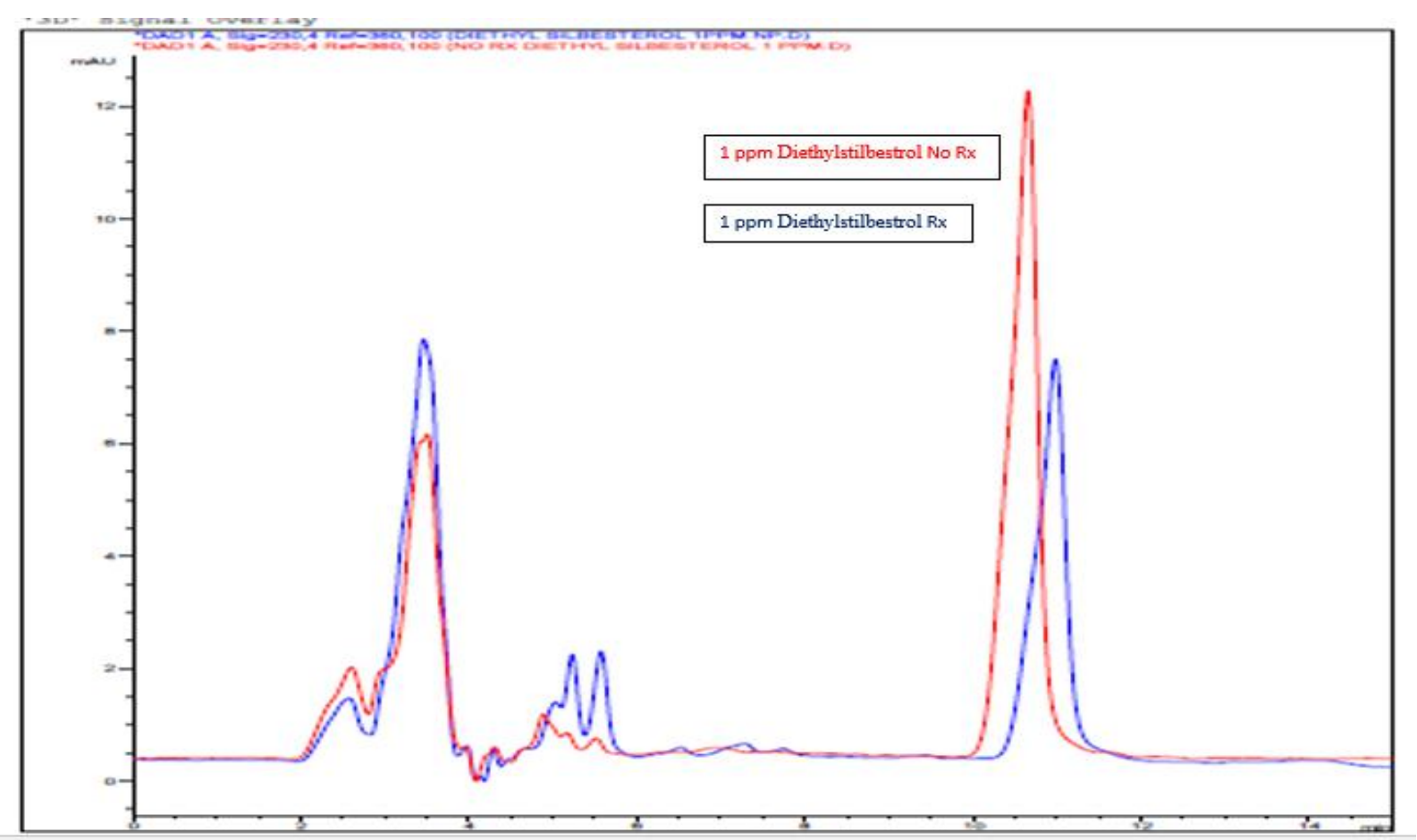

Fig. 13: Overlaid Chromatogram of 1ppm Diethylstilbestrol shows changes in peak area with treatment of AgNP's 


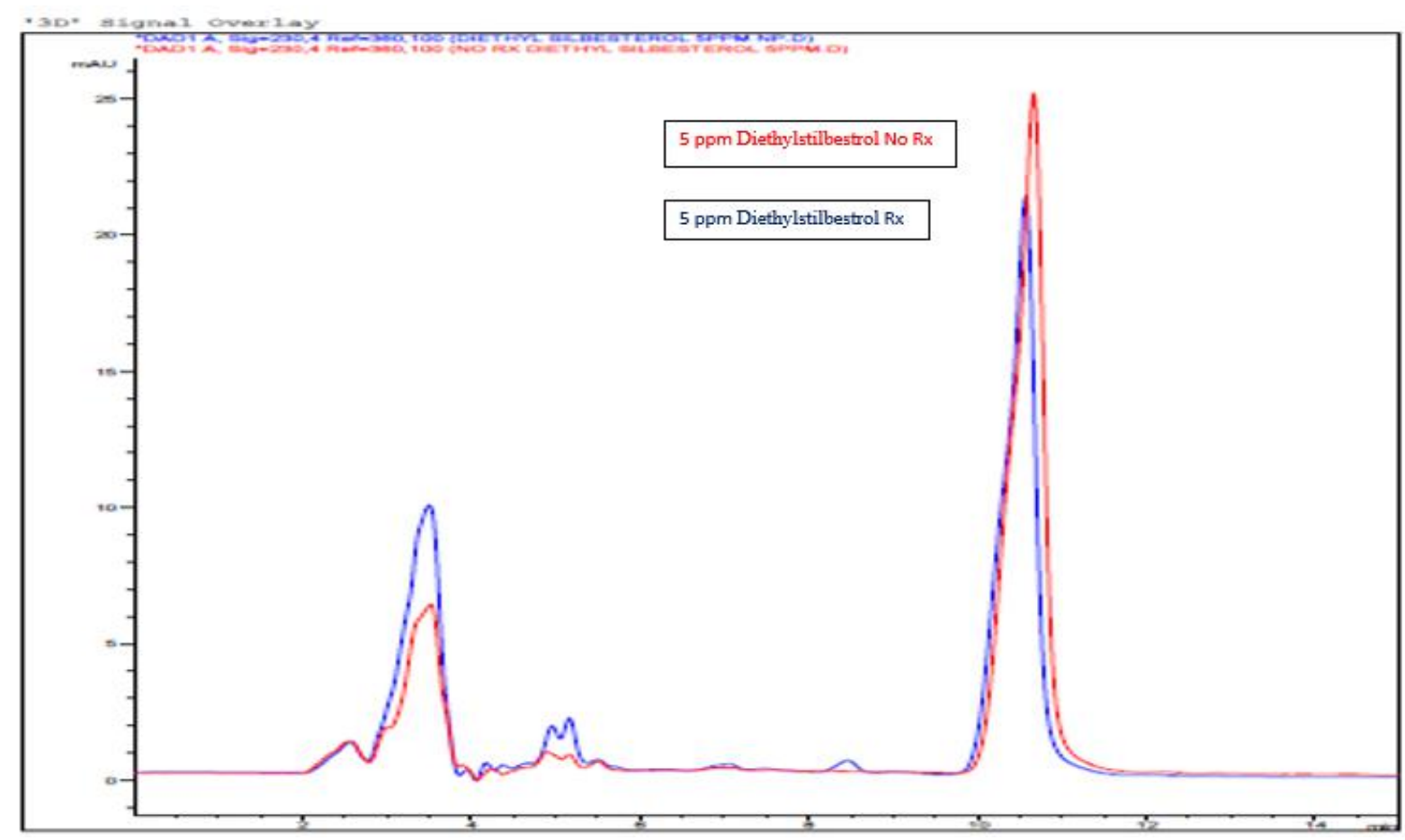

Fig. 14: Overlaid Chromatogram of 5 ppm Diethylstilbestrol shows changes in peak area with treatment of AgNP's

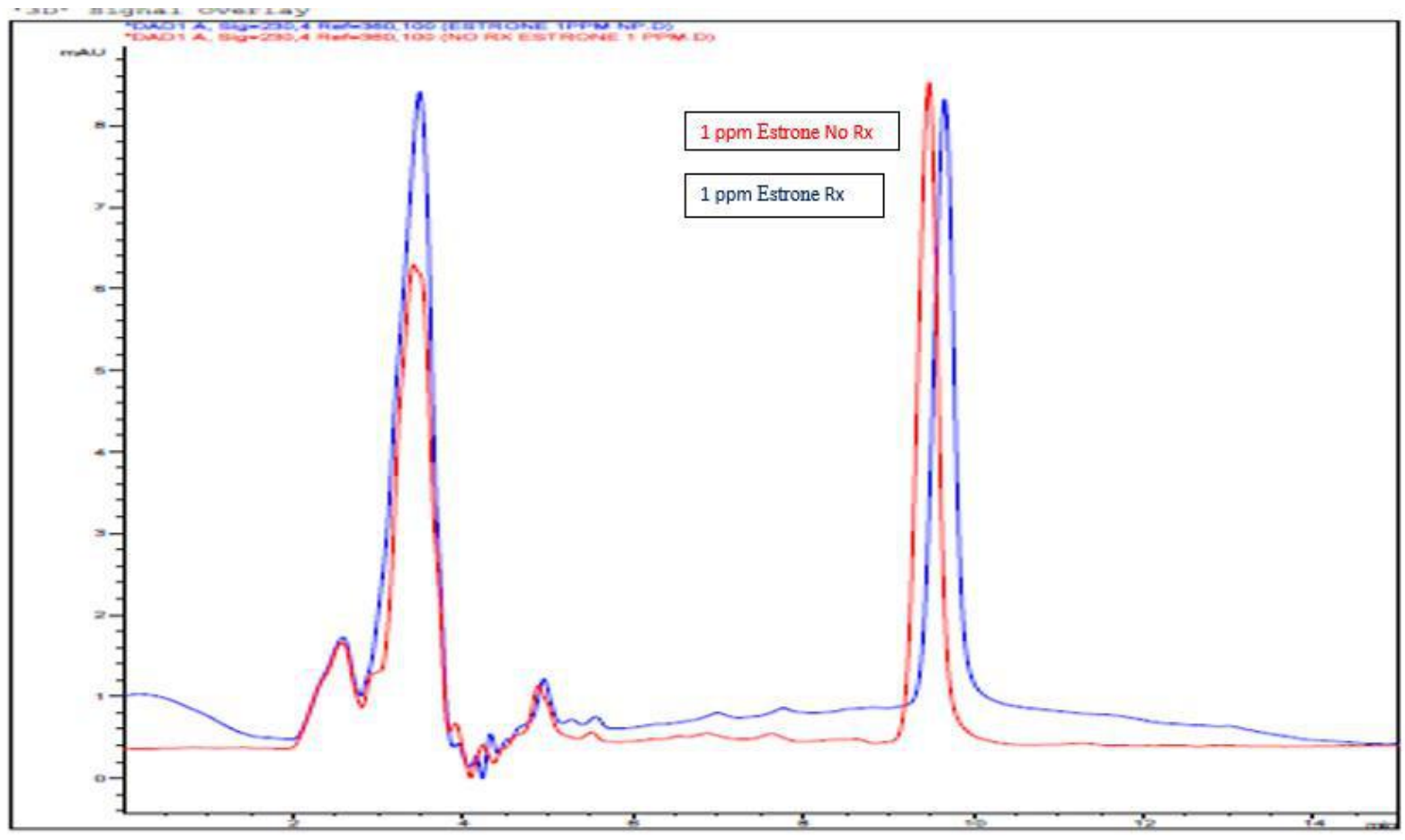

Fig. 15: Overlaid Chromatogram of 1ppm Estrone shows changes in peak area with treatment of AgNP's 




Fig. 16: Overlaid Chromatogram of 5ppm Estrone shows changes in peak area with treatment of AgNP's

Table. 1: Summary of changes in peak areas for selected hormones before and after treatment with AgNP's (-ve sign indicates decrease in peak area (percentage))

\begin{tabular}{|l|l|l|l|l|l|}
\hline S.NO & Compound Name & $\begin{array}{l}\text { 1 ppm } \\
\text { (Area) }\end{array}$ & $\begin{array}{l}\mathbf{5} \text { ppm } \\
\text { (Area) }\end{array}$ & $\begin{array}{l}\text { 1 ppm EDC Rx with 10 } \\
\text { PPM NP's (Area) }\end{array}$ & $\begin{array}{l}\text { 5 ppm EDC Rx with 10 } \\
\text { PPM NP's (Area) }\end{array}$ \\
\hline 1 & $\begin{array}{l}\text { 17 Alpha ethynyl } \\
\text { estradiol (Rt= 8.6) }\end{array}$ & 116 & 660 & 133 & $644(-2.3 \%)$ \\
\hline 2 & $\begin{array}{l}\text { Beta Estradiol } \\
(\text { Rt= 7.6) }\end{array}$ & 153 & 519 & $138(-10.4 \%)$ & 539 \\
\hline 3 & $\begin{array}{l}\text { Estrone } \\
(\text { Rt=9.4) }\end{array}$ & 138 & 244 & $129(-6.5 \%)$ & 292 \\
\hline 4 & $\begin{array}{l}\text { Diethylstilbestrol } \\
(\text { Rt= 10.6) }\end{array}$ & 280 & 653 & $177(-36.7 \%)$ & $552(-15.5 \%)$ \\
\hline 5 & $\begin{array}{l}\text { 19 Norethindrone } \\
(\text { Rt= 8.5) }\end{array}$ & 334 & 1,597 & $300(-10.2 \%)$ & $1,586(-0.64 \%)$ \\
\hline 6 & $\begin{array}{l}\text { D Norgestrel } \\
(\text { Rt= 11.6) }\end{array}$ & 344 & 874 & $316(-8 \%)$ & $630(-28 \%)$ \\
\hline
\end{tabular}

